\title{
Site specific expression of CXCL-I $2 \beta$ and its regulation by miR-200a in ulcerative colitis
}

\begin{abstract}
Purpose: CXCL-12 $\beta$ is a chemokine playing an important role in inflammation and cancer. Altered cytokine and chemokine expression is a hallmark of ulcerative colitis. Emerging evidence suggest that microRNAs are involved in inflammatory process and cancer development during chronic inflammation. This study was done to find the regulation of CXCL-12 expression via different miRNAs in UC.
\end{abstract}

Methods: Biopsy samples were collected from UC patients and non-IBD controls. CXCL$12 \beta$ expression was analyzed at mRNA as well as protein level in UC and compared to controls. Expression of miRNAs targeting CXCL-12 $\beta$ was analyzed by microarray and real time PCR. In vitro studies were done to validate CXCL-12 $\beta$ as a target of miR-200a-3p.

Results: Expression of CXCL-12 $\beta$ was increased in UC compared to controls. MiR-141$3 p$ and miR-200a-3p were predicted to target CXCL-12 $\beta$. Expression of miR-141-3p was upregulated and expression of miR-200a was downregulated in UC compared to controls. In vitro studies using reporter assay proved CXCL-12 $\beta$ as a target miR-200a. Expression of CXCL-12 $\beta$ increased while expression of mir-200a decreased in the cancer prone, rectosigmoid area of colon.

Conclusion: Our study reveals that miR-200a-3p regulates the expression of CXCL-12 $\beta$ in UC. Both CXCL-12 $\beta$ and miR-200a also show site specific altered expression in the colon of UC patients. Our data shows that regulation of CXCL-12 $\beta$ expression using miRNA may be a promising therapeutic approach in UC pathogenesis.

Keywords: Ulcerative Colitis, Cancer, MiRNA, CXCL12
Volume 9 Issue 6 - 2018

\author{
Raju Ranjha,' Surbhi Aggarwal,' Vineet Ahuja,' \\ Jaishree Paul' \\ 'School of Life Sciences, Jawaharlal Nehru University, India \\ ${ }^{2}$ Department of Gastroenterology, All India Institute of Medical \\ Sciences, India
}

Correspondence: Dr. Jaishree Paul, School of Life Sciences, Jawaharlal Nehru University, New Delhi, I I0067, India, Email.jpaul33@hotmail.com

Received: May 15, 2018 | Published: December 31, 2018

\section{Introduction}

IBD is a chronic inflammatory disease of gastrointestinal tract which involve complex interactions between immune system, genetic makeup, gut microbiota and environmental factors. ${ }^{1}$ Ulcerative Colitis (UC) is one of the two main subtypes of Inflammatory Bowel Disease (IBD). ${ }^{2}$ Along with other pathogenic factors, altered cytokine and chemokine expression play important role in the pathogenesis of UC. ${ }^{3,4}$ Patients with prolonged UC have increased risk of developing colorectal cancer (CRC)..$^{5-8}$

Expression of pro-inflammatory cytokines and chemokines are known to increase in UC. ${ }^{3,9}$ The degree of inflammation and tissue damage is dependent on the concentration of local chemokines. Thus over expression of chemokines leads to enhanced recruitment of immune cells to the inflammatory site. ${ }^{9}$ Experimental evidences show that CXCR4/CXCR7/CXCL12 chemokine axis play crucial role in inflammation as well as cancer. CXCL12/CXCR4 chemokine axis is known to be involved in pathogenesis of IBD specially UC. ${ }^{10}$ CXCL12 is expressed by normal intestinal epithelial cells (IECs) and upregulated in inflammed IECs of IBD patients. ${ }^{11}$ Interestingly, enhanced expression of CXCL-12 was observed in UC compared to $\mathrm{CD}$ and more specifically in inflammed compared to non-inflammed mucosa. ${ }^{12}$ Upregulation of CXCL12 in the inflammed colonic mucosa is specifically due to the variant CXCL12 $\beta$ upregulation. ${ }^{13}$ Polymorphisms in CXCL12 are also reported as risk factor for IBD development. ${ }^{14,15}$ Blockade of CXCL12/CXCR4 axis has been shown to ameliorate colitis in both DSS induced as well as in IL-10 KO mice model of colitis. Antagonist of CXCR4 also showed potent antiinflammatory effect. ${ }^{10}$

Micro RNAs (miRNA) are small non-coding RNA molecules that are emerging key players in pathology of many diseases including IBD and cancer. ${ }^{16,17}$ MiRNA are differentially expressed in colonic mucosa and serum of IBD patients and regulate important genes involved in the disease pathogenesis..$^{11,18,19}$ It has been shown that miRNA plays important role in fine-tuning of TLR signaling and maintaining homeostasis of gut microbiota, two very important players in the pathology of IBD. ${ }^{20,21}$

There is increased risk of cancer development due to chronic inflammation. ${ }^{22,23}$ There is also an enhanced risk of CRC development in IBD patients specially UC. ${ }^{3}$ In UC, cancer development predominantly occurs in the rectosigmoid part of colon. ${ }^{6,25}$ MiR141 regulate the expression of CXCL-12 $\beta$, which further mediate leukocyte migration in murine model of colitis and CD. ${ }^{13}$ MiRNAs are reported as connecting link between inflammation and cancer. $22,23,26$ CXCL12 is a pro-angiogenic chemokine that act as important player in chronic inflammation as well as cancer development. ${ }^{27,28}$ Different miRNAs are reported to regulate the expression of CXCL12 in various cancers. ${ }^{13,}$, 27,29-39 Earlier studies have reported that tumor promoting miRNAs are overexpressed and tumor suppressing miRNAs are dowregulated in cancer prone rectosigmoid part of colon in UC. ${ }^{4}$

In the present study we investigated the regulation of CXCL12 $\beta$ through miRNA in UC. CXCL12 $\beta$ expression was determined in colon biopsy samples as well as serum samples of UC patients and controls. 
The impact of miR-200a over-expression on level of CXCL12 $\beta$ was determined.

\section{Materials and Methods}

Patient Samples and cell lines: $30 \mathrm{UC}$ patients and 25 non-IBD controls were included in this study. 20 samples from UC patients were from rectosigmoid region of colon and 10 were from ascending region of colon. Control samples were non IBD individuals without any IBD symptoms and without any colon inflammation. All control and patient samples were age and sex matched. Diagnosis of UC patients were based on the clinical findings based on ECCO guidelines [5]. UC was confirmed by histopathological analysis of colon biopsy samples. This study was approved by Institutional Ethics Review Board, JNU (REF no. 2012/student/28) and the ethical committee of All India Institute of Medical Sciences, New Delhi, India (Ref no. IEC/NP-320/2012). A written consent was taken from all the participants included in this study. Human colorectal adenocarcinoma cell line, HT-29 (National Center for Cell Sciences, Pune, India) were cultured in Dulbecco's Modified Eagle's medium (Gibco, Thermo Fisher Scientific, USA) for in vitro studies.

RNA isolation and RNA quality check: mirVana miRNA isolation kit (Ambion INC, TX 78744, USA) was used for total RNA isolation. RNA was stored at $-80^{\circ} \mathrm{C}$ until further analysis. RNA quality was assessed by running it on $1.5 \%$ agarose gel for evaluating the three bands $28 \mathrm{~S}, 18 \mathrm{~S}$ and $5 \mathrm{~S}$ rRNA. RIN value for the total RNA was calculated using bioanalyser (Agilent Techonologies, California, USA). Samples with RIN value more than 7 were included for microarray analysis.

Microarray Analysis: MiRNA microarray was performed by Exiqon A/S, Denmark via miRCURY LNA ${ }^{\mathrm{TM}}$ microRNAArray ( 7 th gen). Five RNA samples were pooled in each category in equal concentration. ${ }^{6}$ The array data has been submitted in NCBI Gene Expression Omnibus (GEO) under the entry series GSE66932.

MiRNA target prediction and qRT-PCR: Target prediction for miRNAs targeting 3'UTR of CXCL12 $\beta$ was done using online target prediction tools ( Targetscan, Diana-microT and PicTar). RNA was reverse transcribed to cDNA using miRNA specific stem loop primers for miRNAs ${ }^{7}$ and Random Hexamer primers were used for cDNA synthesis for other genes by revert aid cDNA synthesis kit (Fermentas St. Leon Rot, Germany). Table 1 shows the primers sequences used for reverse transcription and real time PCR analysis. Real time PCR was performed using ABI PRISM 7500 Real time PCR system (Applied Biosystems). U6 snRNA and GAPDH were used as internal controls. Parameters for real time were- initial denaturation- $94^{\circ} \mathrm{C}$ for $2 \mathrm{~min}$, denaturation- $94^{\circ} \mathrm{C}$ for $30 \mathrm{sec}$, annealing- $60^{\circ} \mathrm{C}$ for $1 \mathrm{~min}$ for 40 cycles. $20 \mu \mathrm{l}$ reaction containing $7 \mu \mathrm{MQ}$ water, $10 \mu \mathrm{l}$ SYBER Green universal PCR Master Mix (Applied Biosystems, California, USA), $1 \mu \mathrm{l}$ of each forward and reverse primer $(4$ picomole $/ \mu \mathrm{l}), 1 \mathrm{ul}$ cDNA. The relative expression was calculated using the equation $2^{-\Delta \Delta \mathrm{CT}}$ where $\Delta \Delta \mathrm{CT}=\Delta \mathrm{CT}$ target $-\Delta \mathrm{CT}$ control.

Table I Sequence of primers used in Reverse Transcription and qRTPCR.*

\begin{tabular}{|c|c|c|}
\hline \multicolumn{2}{|l|}{ Name } & Primer $\left(5^{\prime}-3^{\prime}\right)$ \\
\hline \multicolumn{2}{|c|}{ Reverse Universal Primer for miRNA } & GTGCAGGGTCCGAGGT \\
\hline \multirow{2}{*}{ hsa-miR-|4|-3p } & RT Primer & GTCGTATCCAGTGCAGGGTCCGAGGTATTCGCACTGGATACGCCATCTTT \\
\hline & Forward & TAACACTGTCTGGTAAAGAT \\
\hline \multirow{2}{*}{ hsa-miR-200a-3p } & RT Primer & GTCGTATCCAGTGCAGGGTCCGAGGTATTCGCACTGGATACGACATCGTT \\
\hline & Forward & TAACACTGTCTGGTAACGAT \\
\hline \multirow{2}{*}{ CXCL-I $2 \beta$} & Forward & ССTGCCACAGCСTССССТ \\
\hline & Reverse & TAAGCTGCTACGTGTCGCC \\
\hline
\end{tabular}

* RT primers were used for cDNA synthesis in each case; Reverse universal and forward primers were used for real time analysis for each miRNA

Cloning and transfection: miR-200a was cloned in pBABEpuro vector. DNA fragment containing sequence of miR200a and its flanking region was used for cloning. Primers used for miR-200a cloning were Forward miR-200a 5'CGCGGATCCACAGCCATCTTCCCTCCTG- 3' and Reverse miR200a 5'- CCGGAATTCGGAGCTGACACAGGCCCTC- $3{ }^{\prime} .{ }^{8} 3$ 'UTR of CXCL12 $\beta$ was cloned in pMIR-Report vector downstream of luciferase gene. Primers used for cloning were Forward CXCL12 $\beta$ 5'-CGAGCTCGGGTCAGACGCCTGAGGAAC-3' and Reverse CXCL12 3 , -CGACGCGTACATTTGAGTACAGAACTTTATT3'. Transfection of plasmids was done using Lipofectamine 3000 (Thermofisher Scientific, USA) following manufacturer's protocol.

Enzyme Linked Immunosorbant Assay (ELISA): CXCL-12 $\beta$ concentration in the patient serum samples and cell culture supernatant was measured using ELISA kit (Sunred Biological, Shanghai). The assay was performed according to manufacturer's protocol and data was analysed using curve expert 1.4 software.

Luciferase assay: Luciferase assay was performed using Dual luciferase assay kit (Promega, USA). PRL-TK vector was used for normalization. CXCL12 3 3'UTR containing luciferase vector was transfected in HT-29 cells with or without miR-200a overexpression.

Statistical analysis: Statistical analysis was done using two-way student's t test. Data analysis was done using Graphpad Prism 5.0. A probability level of $\mathrm{p}<0.05$ was considered statistically significant.

\section{Results}

CXCL12 $\beta$ expression in UC vs Control individuals: CXCL12 $\beta$ expression was analyzed both at mRNA as well as protein level. There was significant increase in CXCL12 $\beta$ expression in biopsy samples from UC patients compared to controls (Figure 1a). Level of CXCL12 $\beta$ was increased in the serum samples of UC patients compared to non IBD controls (Figure1b).

MiRNAs targeting 3'UTR of CXCL12ß: Target prediction analysis showed miR-141-3p and miR-200a, members of miR200 family, targeting 3'UTR of CXCL12 $\beta$. Both target same two sites, position 51-58 and 2940-2948 in the 3'UTR of CXCL12 $\beta$ (http://www.targetscan.org/cgi-bin/targetscan/vert_71/view_ gene. $\quad$ cgi? $\quad$ rs $=$ ENST00000374429.2\& taxid $=9606 \&$ member $\mathrm{s}=\&$ showcnc $=0$ \& shownc $=0$ \& showncf $1=\&$ showncf $2=\&$ subset $=1$ ). 
Figure 1a

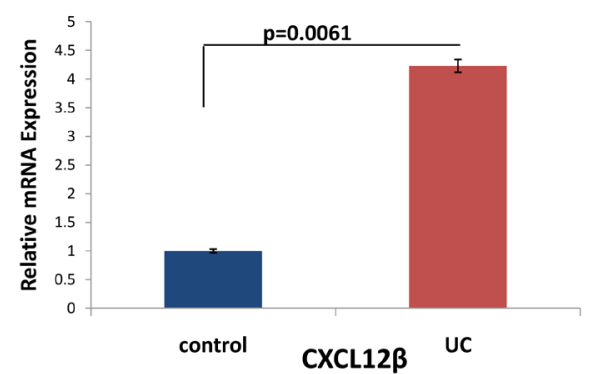

Figure 1b

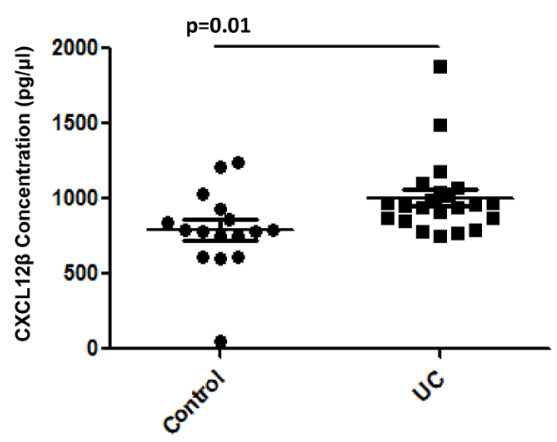

Figure I Increased expression of CXCLI $2 \beta$ in UC: a) Total RNA was isolated from colon biopsy samples from healthy individuals and UC patients and reverse transcribed to cDNA using random hexamer primers. Data derived from quantitative real-time PCR. Normalization was performed with the GAPDH. Sample size was $n=25$ for $U C$ and $n=25$ for controls. There was significant increase in expression of CXCLI2 $\beta$ in UC patients compared to controls. Bars represent Standard Error of mean (SEM). b) Concentration of CXCLI2 $\beta$ measured in human serum samples by ELISA, $n=25$ in each category. There was significant increase in serum concentration of CXCLI2 $\beta$ in UC patients compared to controls.

Expression of miR-141-3p and miR-200a: miRNAs expression was analysed in UC compared to non-IBD controls using microarray analysis. Figure 2 represents the heatmap for the microarray data describing the expression profile of miRNAs in UC and non-IBD controls. Expression of both the miRNAs was decreased in UC compared to control. Microarray data was validated using qRTPCR. MiR-141-3p showed upregulation in our qRTPCR analysis in contrary to microarray data (Figure 3a). Expression of miR-200a however decreased significantly in UC patients compared to controls in qRTPCR results similar to microarray data (Figure $3 b$ ).

In vitro validation of CXCL12 $\beta$ as a target of miR-200a: In vitro validation was carried out by miR-200a overexpression and reporter assay.

Effect of miR-200a overexpression on CXCL12 $\beta$ secretion: We looked at the effect of miR-200a overexpression on CXCL12 $\beta$ secretion in HT-29 cell line. Secretion of CXCL12 $\beta$ decreased in the supernatant of HT-29 cells overexpressing miR-200a compared to control transfected and non-transfected cells (Figure 4).

Dual Luciferase assay: Further validation of CXCL12 $\beta$ as miR-200a target was done using 3'UTR reporter gene assay. Luciferase was cloned upstream of 3'UTR of CXCL12 $\beta$ and was used as a reporter. So the luciferase activity was controlled by miR-200a. The level of luciferase activity was decreased by overexpression of miR-200a (Figure 5).

Site specific expression of miR-200a and CXCL12 $\beta$ in colon of UC patients: We looked at the site specific expression of CXCL12 $\beta$ and miR-200a in rectosigmoid vs ascending colon of UC patients. Expression of miR-200a and CXCL12 $\beta$ showed contrasting pattern of expression. Expression of CXCL12 $\beta$ was increased in rectosigmoid colon in UC patients compared to controls. Expression of the chemokine in ascending colon was comparable to non-IBD controls (Figure 6a). Expression of miR-200a was decreased in rectosigmoid colon compared to ascending colon of UC patients and to non-IBD controls (Figure 6b).

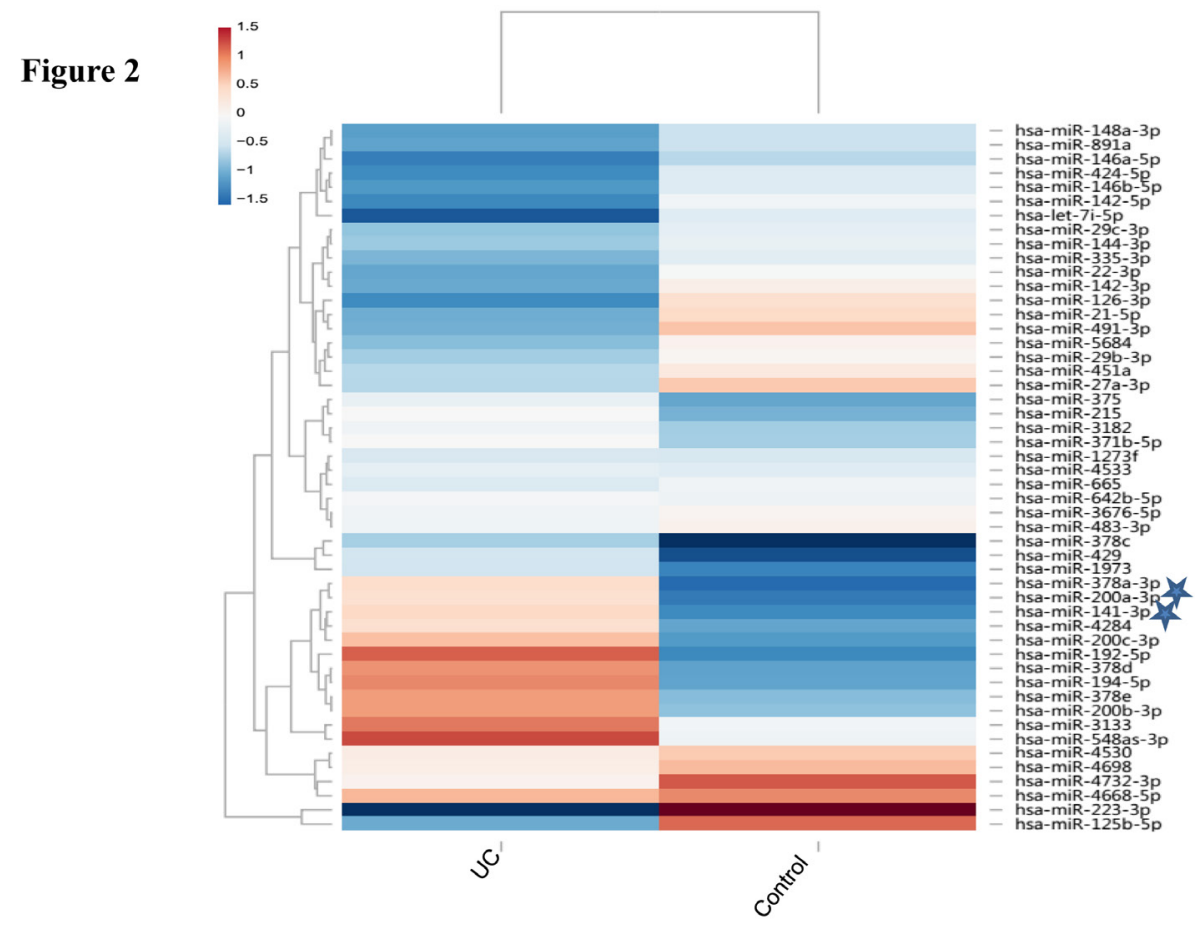

Figure 2 Heatmap and unsupervised hierarchical clustering of miRNAs. Heatmap showing differential miRNA expression in UC patients vs Controls. 
Figure 3a

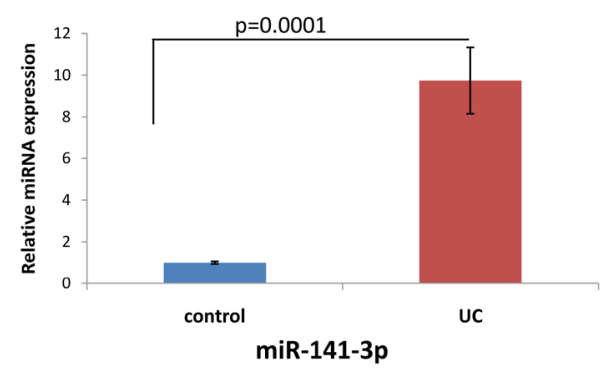

Figure 3b

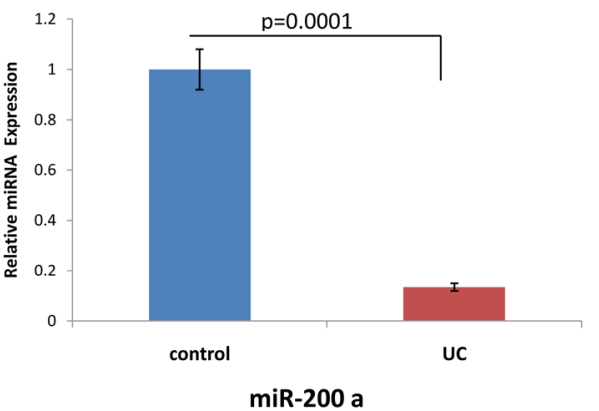

Figure 3 Relative Expression of CXCL- $12 \beta$ targeting miRNAs. Total RNA was isolated from colon biopsy samples from healthy subjects and UC patients and reverse transcribed to cDNA using specific primers. Data derived from quantitative real-time PCR. Normalization of samples was performed with the small nuclear U6 snRNA. a) Relative expression of miR-14I-3p in UC vs controls. b) Relative Expression of miR-200a-3p in UC vs controls. $n=25$ for each category. Bars represent SEM.

Figure 4

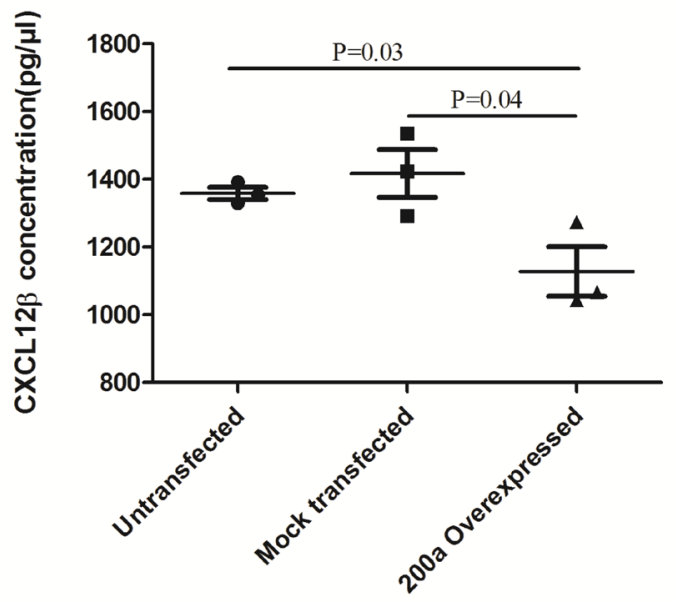

Figure 4 Effect of miR-200a-3p overexpression on CXCL-I2 $\beta$ secretion. miR-200a gene was cloned in PBABE-puro vector and transfected in HT-29 cell line. Untransfected cells and mock transfected cell were taken as controls. Cell supernatant was collected after $48 \mathrm{hrs}$ of transfection and CXCL-I2 $\beta$ concentration was measured using ELISA. Bars represent SEM.

\section{Discussion}

CXCL12 $\beta$ is reported to play important role in chronic inflammation and various type of cancers. ${ }^{12,27,28}$ Different miRNAs were reported to regulate CXCL12 expression in different diseases. . $^{13,27,29-39}$ In this study we investigated how the expression of CXCL12 $\beta$ is regulated by miRNA during UC. We also looked at site specific expression of
CXCL12 $\beta$ and its targeting miRNA in context to CRC susceptibility of various parts of colon.

CXCL12 is a constitutive cytokine reported to play important role in various inflammatory diseases including lung, brain, joint and intestinal inflammation. CXCL12 is constitutively expressed by colon epithelial cells. Its expression was reported to be increased in IBD. Its expression is more pronounced in UC compared to CD and in inflamed mucosa compared to non-inflamed mucosa. CXCL12CXCR4 axis contributes to intestinal epithelial cells migration, barrier function, restitution and cAMP mediated cellular functions in normal intestinal mucosa. ${ }^{44,45}$ Increased CXCL12 expression attracts immune cells which infiltrate and secrete proinflammatory cytokines in inflamed mucosa. ${ }^{13}$ CXCL-12 has different splice variants. Due to four additional residues at its C-terminus, CXCL- $12 \beta$ variant is found more resistant to proteolysis. It can maintain a more potent concentration in the blood in order to recruit more leukocytes. ${ }^{46,47}$ Increased expression of CXCL12 in colonic mucosa is mainly contributed by increased expression of CXCL12 $\beta .{ }^{13} \mathrm{We}$ looked at the expression of CXCL12 $\beta$ in UC vs non-IBD controls. We also found increased expression of CXCL12 $\beta$ at mRNA level in colonic mucosa (Figure 1a) and at protein level in the serum (Figure 1b) of UC patients compared to non-IBD controls.

We looked for the miRNAs targeting 3'UTR of CXCL12 $\beta$. It was predicted to be target of miR-141-3p and miR-200a. Both are member of miR-200 family and target same site in the 3'UTR of CXCL12 3 . Expression of both miR-141-3p and miR-200a was decreased in our microarray analysis (Figure 2). We looked at the miR-141-3p expression in the colon of UC patients and controls. MiR-141-3p was reported to regulateCXCL12 $\beta$ expression in the colon of CD patients. ${ }^{13}$ Expression of miR-141-3p was increased in the colon of UC patients compared to non-IBD controls in our real time analysis (Figure 3a). As miR-141-3p showed same expression as that of target gene, it revealed that probably miR-141-3p was not regulating the expression of CXCL-12 $\beta$ in case of UC. Our real time expression data was in disagreement with our microarray data. Microarray data was often reported to show disagreement with real time analysis. ${ }^{9}$ We next did real time PCR to validate the expression of miR-200a-3p. Real time expression analysis showed that there was a significant decrease in the expression of miR-200a-3p (Figure $3 b$ ), as found in microarray analysis. This indicated that miR-200a-3p might be regulating the expression of CXCL-12 $\beta$ in UC.

MiR-200a-3p was transfected and overexpressed in HT-29 cell line. Increase in expression of miR-200a-3p resulted in significant decrease in secretion of CXCL-12 $\beta$ from HT-29 cell line (Figure 4). We performed dual luciferase assay in order to confirm if the regulation of CXCL-12 $\beta$ is mediated by miR-200a-3p. Increase in expression of miR-200a-3p leads to significant decrease in luciferase activity (Figure 5)

During the process of intestinal inflammation, increased level of CXCl-12 $\beta$ attract different immune cells, which further secrete a large volume of pro-inflammatory cytokines. CXCL12 is a constitutive and inflammatory chemokine in the intestinal immune system. Selective manipulation of the CXCL12/CXCR4 axis had been suggested as potential therapeutic mechanism in IBD. ${ }^{13}$ Blockade of CXCL12/ CXCR4 Axis ameliorates murine colitis in experimental mice model of colitis. ${ }^{10}$ It has been hypothesized that the CXCL12 $\beta$ targeted by miR-141 in colonic epithelial cells serves as an important mechanism regulating colonic CXCL12 $\beta /$ total-CXCL12 expression and control immune cell trafficking during both experimental colitis models 
and the $\mathrm{CD}$ dysfunction process in humans. Our expression data in patients and validation by in vitro studies proves our hypothesis that the expression of miR-200a-3p regulates the level of CXCL-12 $\beta$ chemokine in the colonic cells during ulcerative colitis.

\section{Figure 5}

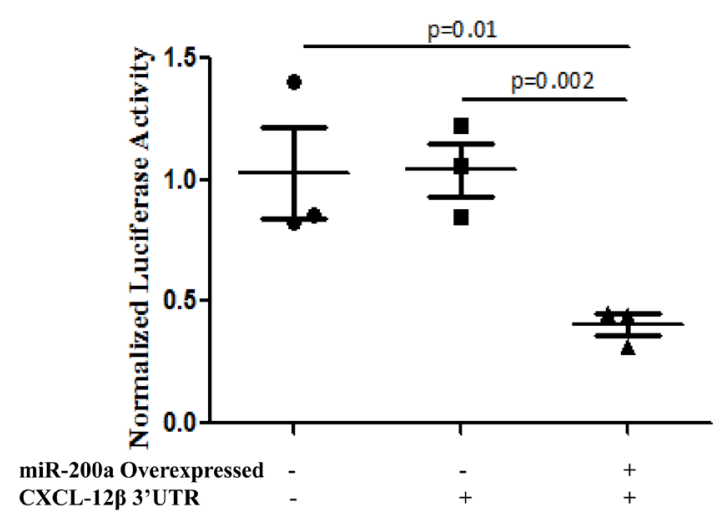

Figure 5 Dual Luciferase assay. 3'UTR reporter gene assay was performed to confirm CXCL-I $2 \beta$ as a target of miR-200a-3p. Luciferase was cloned upstream of 3'UTR of CXCLI $2 \beta$ and used as a reporter. Effect of miR-200a$3 p$ overexpression was observed by measuring the luciferase activity. Bars represent SEM.

Figure 6a

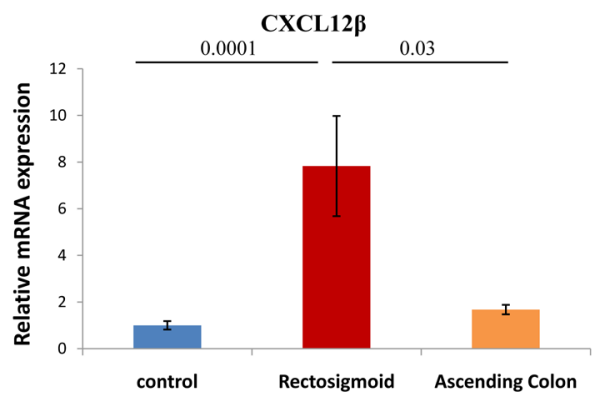

Figure 6b

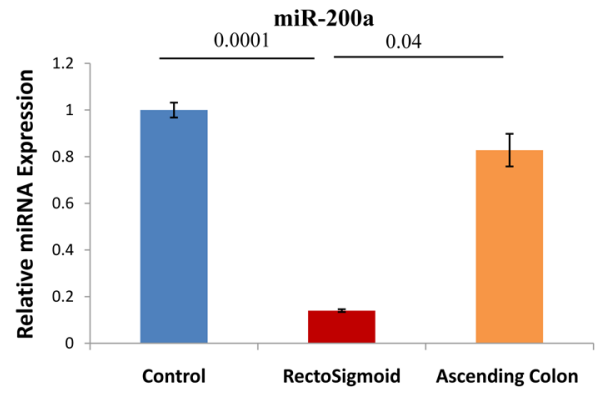

Figure 6 Site specific expression of CXCL-I2 $\beta$ and its targeting miRNA in colon. Expression of (a) CXCL-I $2 \beta$ (b) miR-200a was analyzed in Ascending colon and Rectosigmoid part of colon of UC patients. $n=25$ for controls, $n=20$ for Rectosigmoid and $n=10$ for Ascending Colon. Bars represent SEM.

Rectosigmoid part of the colon of UC patients was reported to be cancer prone in inflammation associated carcinoma development. MiRNA expression was also altered in the rectosigmoid part of colon compared to ascending colon in UC. ${ }^{4}$ Proinflammatory CXCL12CXCR4 signaling axis has been reported to play important role in cancer development. ${ }^{10}$ We looked at the site specific expression of CXCL12 $\beta$ and its targeting miRNA. There was significant increase in expression of CXCL12 $\beta$ and the expression of miR-200a decreased significantly in the rectosigmoid part of colon compared to ascending colon and non-IBD controls (Figure 6a and 6b). This further strengthen our analysis that miR-200a regulate the expression of CXCL12 $\beta$ in UC and do so in a site specific manner. It also showed that CXCL12 may be an important factor in UC associated CRC development as it exhibited increased expression in the cancer prone rectosigmoid part of colon.

\section{Conclusion}

In conclusion our study show regulation of CXCL-12 $\beta$ in UC is mediated by miR-200a, not by miR-141-3p as in CD. Our results further revealed that miR-200a was predominantly decreased in the colonic epithelial cells of UC patients leading to increase in expression of CXCL-12 $\beta$ in the colon. Both CXCL12 $\beta$ and its targeting miRNA showed site specific expression in UC. Inhibiting CXCL-12 $\beta$ expression using miRNA may prove to be a valuable approach for treatment of IBD and to prevent IBD associated cancer development.

\section{Acknowledgments}

The current work was financially supported by the Department of Biotechnology, New Delhi DBT grant (award no. BT/PR8348/ MED/30/1023/2013 awarded to JP. We acknowledge umbrella funding from DST PURSE II : File No. 6(54)/SLS/JP/DST PURSE/2016-17 awarded to JP. We thank the participants who contributed samples for this study. RR gratefully acknowledges the research fellowship from Council of Scientific and Industrial Research, New Delhi

\section{Conflicts of interest}

The authors declare that no conflicts of interest.

\section{References}

1. Ananthakrishnan AN. Epidemiology and risk factors for IBD. Nat Rev Gastroenterol Hepatol. 2015;12(4):205-217.

2. Ahuja V. Inventory of a reservoir: friends \& foes. Indian $J$ Med Res. 2015;142(1):4-6.

3. Neurath MF. Cytokines in inflammatory bowel disease. Nat Rev Immunol. 2014;14(5):329-342.

4. Xavier RJ, Podolsky DK. Unravelling the pathogenesis of inflammatory bowel disease. Nature. 2007;448(7152):427-434.

5. Nowacki TM, Bruckner M, Eveslage M, et al. The risk of colorectal cancer in patients with ulcerative colitis. Dig Dis Sci. 2015;60(2):492501.

6. Ranjha R, Aggarwal S, Bopanna S, et al. Site-Specific MicroRNA Expression May Lead to Different Subtypes in Ulcerative Colitis. PLoS One. 2015;10(11):e0142869.

7. Ullman TA, Itzkowitz SH. Intestinal inflammation and cancer. Gastroenterology. 2011;140(6):1807-1816.

8. Zhang L, Fan XM. The pathological role of microRNAs and inflammation in colon carcinogenesis. Clin Res Hepatol Gastroenterol. 2015;39(2):174-179.

9. Banks C, Bateman A, Payne R, et al. Chemokine expression in IBD. Mucosal chemokine expression is unselectively increased in both ulcerative colitis and Crohn's disease. J Pathol. 2003;199(1):28-35.

10. Mikami S, Nakase H, Yamamoto S, et al. Blockade of CXCL12/CXCR4 axis ameliorates murine experimental colitis. J Pharmacol Exp Ther. 2008;327(2):383-392.

11. Feng $\mathrm{X}$, Wang $\mathrm{H}$, Ye $\mathrm{S}$, et al. Up-regulation of microRNA-126 may contribute to pathogenesis of ulcerative colitis via regulating NF-kappaB inhibitor IkappaBalpha. PLoS One. 2012;7(12):e52782. 
12. Dotan I, Werner L, Vigodman S, et al. CXCL12 is a constitutive and inflammatory chemokine in the intestinal immune system. Inflamm Bowel Dis. 2010;16(4):583-592.

13. Huang Z, Shi T, Zhou Q, et al. miR-141 Regulates colonic leukocytic trafficking by targeting CXCL12beta during murine colitis and human Crohn's disease. Gut. 2014;63(8):1247-1257.

14. Mrowicki J, Przybylowska K, Dziki L, et al. Association of the$801 \mathrm{G} /$ A polymorphism of CXCL12 gene with the risk of inflammatory bowel diseases development in a Polish population. Pol Przegl Chir 2011;83(6):334-338.

15. Mrowicki J, Przybylowska-Sygut K, Dziki L, et al. The role of polymorphisms of genes CXCL12/CXCR4 and MIF in the risk development IBD the Polish population. Mol Biol Rep. 2014;41(7):4639 4652

16. Winter J, Jung S, Keller S, et al. Many roads to maturity: microRNA biogenesis pathways and their regulation. Nat Cell Biol. 2009;11(3):228234

17. Ranjha R, Meena NK, Singh A, et al.. Association of miR-196a-2 and miR-499 variants with ulcerative colitis and their correlation with expression of respective miRNAs. PLoS One. 2017;12(3):e0173447.

18. Wang $\mathrm{H}$, Chao $\mathrm{K}, \mathrm{Ng} \mathrm{SC}$, et al. Pro-inflammatory miR-223 mediates the cross-talk between the IL23 pathway and the intestinal barrier in inflammatory bowel disease. Genome Biol. 2016;17:58

19. $\mathrm{Wu} \mathrm{W}, \mathrm{He} \mathrm{C}$, Liu $\mathrm{C}$, et al. miR-10a inhibits dendritic cell activation and Th1/Th17 cell immune responses in IBD. Gut. 2015;64(11):1755-1764.

20. Liu S, da Cunha AP, Rezende RMet al. . The Host Shapes the Gut Microbiota via Fecal MicroRNA. Cell Host Microbe. 2016;19(1):32-43

21. O'Neill LA, Sheedy FJ, McCoy CE. MicroRNAs: the fine-tuners of Toll-like receptor signalling. Nat Rev Immunol. 2011;11(3):163-175.

22. Iliopoulos D. MicroRNA circuits regulate the cancer-inflammation link. Sci Signal. 2014;7(318):pe8.

23. Ranjha R, Paul J. Micro-RNAs in inflammatory diseases and as a link between inflammation and cancer. Inflamm Res. 2013;62(4):343-355.

24. Jones R SM, Cheng J. The Risk of Colon Cancer in Inflammatory Bowel Disease. J Gastroint Dig Syst. 2014;4:164.

25. Choi PM, Zelig MP. Similarity of colorectal cancer in Crohn's disease and ulcerative colitis: implications for carcinogenesis and prevention. Gut. 1994;35(7):950-954.

26. Josse C, Bours V. MicroRNAs and Inflammation in Colorectal Cancer. Adv Exp Med Biol. 2016;937:53-69.

27. Chattopadhyay E, Singh R, Ray A, et al. Expression deregulation of mir31 and CXCL12 in two types of oral precancers and cancer: importance in progression of precancer and cancer. Sci Rep. 2016;6:32735.

28. Noort AR, van Zoest KP, Weijers EM, et al. NF-kappaB-inducing kinase is a key regulator of inflammation-induced and tumour-associated angiogenesis. J Pathol. 2014;234(3):375-385.

29. Arabanian LS, Fierro FA, Stolzel F, et al. MicroRNA-23a mediates posttranscriptional regulation of CXCL12 in bone marrow stromal cells. Haematologica. 2014;99(6):997-1005.

30. Bijkerk R, van Solingen C, de Boer HC, et al. Silencing of miRNA-126 in kidney ischemia reperfusion is associated with elevated SDF-1 levels and mobilization of Sca-1+/Lin- progenitor cells. Microrna 2014;3(3):144-149.

31. Dong S, Jin M, Li Y, et al. MiR-137 acts as a tumor suppressor in papillary thyroid carcinoma by targeting CXCL12. Oncol Rep. 2016;35(4):21512158 .
32. Fan Y, Xu LL, Shi CY, et al. MicroRNA-454 regulates stromal cell derived factor-1 in the control of the growth of pancreatic ductal adenocarcinoma. Sci Rep. 2016;6:22793.

33. Lv Y, Lei $\mathrm{Y}, \mathrm{Hu} \mathrm{Y}$, et al. miR-448 negatively regulates ovarian cancer cell growth and metastasis by targeting CXCL12. Clin Transl Oncol. 2015;17(11):903-909.

34. Pillai MM, Yang X, Balakrishnan I, et al. MiR-886-3p down regulates CXCL12 (SDF1) expression in human marrow stromal cells. PLoS One. 2010;5(12):e14304.

35. Sipert CR, Morandini AC, Dionisio TJ, et al. In vitro regulation of CCL3 and CXCL12 by bacterial by-products is dependent on site of origin of human oral fibroblasts. J Endod. 2014;40(1):95-100.

36. Tamaru S, Mizuno Y, Tochigi H, et al. MicroRNA-135b suppresses extravillous trophoblast-derived HTR-8/SVneo cell invasion by directly down regulating CXCL12 under low oxygen conditions. Biochem Biophys Res Commun. 2015;461(2):421-426.

37. Toritsuka M, Kimoto S, Muraki K, et al. Deficits in microRNAmediated Cxcr4/Cxcl12 signaling in neurodevelopmental deficits in a 22q11 deletion syndrome mouse model. Proc Natl Acad Sci U S A. 2013;110(43):17552-17557.

38. Zernecke A, Bidzhekov K, Noels H, et al. Delivery of microRNA-126 by apoptotic bodies induces CXCL12-dependent vascular protection. $\mathrm{Sci}$ Signal. 2009;2(100):ra81.

39. Zhang J, Liu J, Liu Y, et al. miR-101 represses lung cancer by inhibiting interaction of fibroblasts and cancer cells by down-regulating CXCL12. Biomed Pharmacother. 2015;74:215-221.

40. Dignass A, Eliakim R, Magro F, et al. Second European evidence-based consensus on the diagnosis and management of ulcerative colitis part 1: definitions and diagnosis. J Crohns Colitis. 2012;6(10):965-990.

41. Peng X, Wood CL, Blalock EM, et al. Statistical implications of pooling RNA samples for microarray experiments. BMC Bioinformatics. $2003 ; 4: 26$.

42. Chen C, Ridzon DA, Broomer AJ, et al. Real-time quantification of microRNAs by stem-loop RT-PCR. Nucleic Acids Res. 2005;33(20):e179.

43. Li YX, Liu DQ, Zheng C, et al. miR-200a modulate HUVECs viability and migration. IUBMB Life. 2011;63(7):553-559.

44. Smith JM, Johanesen PA, Wendt MK, et al. CXCL12 activation of CXCR4 regulates mucosal host defense through stimulation of epithelial cell migration and promotion of intestinal barrier integrity. Am J Physiol Gastrointest Liver Physiol. 2005;288(2):G316-326.

45. Werner L, Guzner-Gur H, Dotan I. Involvement of CXCR4/CXCR7/ CXCL12 Interactions in Inflammatory bowel disease. Theranostics. 2013;3(1):40-46.

46. De La Luz Sierra M, Yang F, Narazaki M, et al. Differential processing of stromal-derived factor-1alpha and stromal-derived factor-1beta explains functional diversity. Blood. 2004;103(7):2452-2459.

47. Janowski M. Functional diversity of SDF-1 splicing variants. Cell Adh Migr. 2009;3(3):243-249.

48. Morey JS, Ryan JC, Van Dolah FM. Microarray validation: factors influencing correlation between oligonucleotide microarrays and realtime PCR Biol Proced Online. 2006;8:175-193.

49. Saha A, Ahn S, Blando J, et al. Proinflammatory CXCL12-CXCR4 CXCR7 signaling axis drives Myc-induced prostate cancer in obese mice. Cancer Res. 2017. 